suchungen von BRADLEY ${ }^{16}$ sowie LAPCHINE und ENJALBERT ${ }^{10}$ überein. Sie fanden, daß die terminalen Knöpfe von Phagen der Gruppen A und B ähnliche Struktur besitzen.

Die komplexer aufgebauten Knöpfe von Phagen der Gruppen B und C schienen fester mit dem Schwanz verbunden zu sein als die hexagonale Grundplatte bei Phagen der Gruppe A. Das erklärt vielleicht, weshalb in diesen Untersuchungen Phagen der Gruppe A häufiger ohne terminalen Knopf gefunden wurden. Im Gegensatz dazu war bei Phagen der Gruppen B und C selten ein Verlust des terminalen Knopfes zu beobachten. Darüberhinaus wurden bei Phagen der Gruppe A einige mit längeren Schwänzen gefunden (Abb.4). Die Phagen von Gruppen B und C dagegen besaßen einheitliche

16 D. E. Bradley, J. Roy. microscop. Soc. 84, 257 [1965].

17 E. Boy de la Tour u. E. Kellenberger, Virology 27, 222 [1965].
Schwanzlängen. Man kann daraus schließen, daß es sich bei den Phagen der Gruppe A nicht um abweichende Schwanzlängen handelt, sondern um End zu End-Aggregation von Schwanzbruchstücken. Alle Phagenköpfe besaßen einen hexagonalen Umriß. Daraus konnten aber keine Rückschlüsse über die Oberflächensymmetrie abgeleitet werden. Weitere Untersuchungen müßten klären, ob es sich bei den Köpfen der Staphylokokken-Phagen um Ikosaeder, wie sie bei den T-even Phagen beschrieben wurden ${ }^{17,18}$, oder um Köpfe mit 6-facher Symmetrie in der Längsachse handelt ${ }^{7}{ }^{19}$. Alle T-even Phagen haben hexagonale Grundplatten.

Diese Untersuchungen wurden mit finanzieller Unterstützung des Bundesministeriums für Ernährung, Landwirtschaft und Forsten, Bonn, durchgeführt.

18 M. F. Moody, Virologie 26, 567 [1965].

19 D. E. Bradley, J. gen. Microbiol. 38, 395 [1965].

\title{
Die Trennung der Hämolymphe-Proteine bei Vorpuppen und Puppen der Galleria mellonella L. mittels Gel-Filtration auf Sephadex G-200, Bestimmung ihrer isoelektrischen Punkte und ihrer Molekulargewichte
}

Separation of Haemolymph Proteins of Prepuae and Pupae of Galleria mellonella L. by means of Gel Filtration on Sephadex 200, Determination of their Isoelectric Points and their Molecular Weights

\section{Milan Marek}

Abteilung für Tierphysiologie der Universität J. E. Purkyně's, Brno

(Z. Naturforschg. 24 b, 732-740 [1969]; eingegangen am 27. Dezember 1968)

Gel filtration on Sephadex G-200 was carried out on haemolymph proteins of prepupae, ligatured prepupae, male and female pupae and cooled pupae of Galleria mellonella L.

The proteins were separated into two main fractions. The esterase activity of the eluated haemolymph was determined by means of beta-naphthyl acetate after filtration.

After elution the samples were condensed and additionally separated on horizontal starch-gel electrophoresis.

The "cooling protein" of pupae and the "ligature protein" of ligatured larvae of Galleria mellonella were shown by means of starch-gel electrophoresis to be new proteins, so far not described.

The isoelectric point and molecular weight were determined for the individual protein fractions.

They were then stained with amido black $10 \mathrm{~B}$ for the proof of proteins, and with alpha-naphthyl butyrate with Fast-Blue BB salt for the identification of esterases.

Das Studium der Hämolymphe-Proteine bei den Insekten erfolgte bald nach dem Studium der Proteine der Säugetierseren. Die früheren Studien, unter Verwendung einer chemischen Fraktion, erwiesen die Anwesenheit annähernd gleicher Mengen Albumine $(3,1 \%)$ in der Hämolymphe der Bienenlarve ${ }^{1}$. Wie Kuznecow ${ }^{2}$ anführt ist nach Landois, Poulton

1 G. H. Bishop, A. P. Briggs u. E. J. Ronzoni, J. biol. Chemistry 66, 77 [1925].

2 N. J. Kuznecow, Die Grundlage der Insektenphysiologie. IANSU, Moskau 1948.

${ }^{3}$ L. Landois, E. Poulton u. V. Graber (zitiert von N. J. KUZNECOW, 1948). und GRABER ${ }^{3}$ bei den Insekten vorwiegend Globulin vertreten.

Die Autoren 1.c. ${ }^{4}$ bestimmten im Blute adulter Hydrophilus piceus 2 Albumine und 1 Globulin. Etwa 41 Jahre vorher identifizierte DuCCESCHI ${ }^{5}$ in der Hämolymphe von Bombyx mori auf Grund ihrer Koagulation bei verschiedenen Temperaturen.

${ }^{4}$ M. Florkin u. G. Duchateau, Acta Biol. Belg. 3, 1 [1943].

5 V. Ducceschi, Atti Reale Accad. Georgofili [Firenze] 80, 365 [1902]. 
Seit Einführung der Elektrophorese in die Biochemie wurde die Zusammensetzung der InsektenHämolymphe genauer erkannt ${ }^{6,7}$. Bei den Larven von Galleria mellonella ${ }^{8,9}$ gelang es die Hämolymphe-Proteine mit der Papierelektrophorese in 2 bis 5 Fraktionen zu trennen. Auch Aizawa ${ }^{10}$ unterschied bei den Larven die gleiche Anzahl von Fraktionen, bei den Larven von Drosophila bestimmten Chen ${ }^{11}$, Wunderly und Gloor ${ }^{12} 2$ Proteinfraktionen und $\mathrm{CHEN}^{13}$ bei den Larven vom Culex 1 Fraktion. Schмidt ${ }^{14}$ ermittelte bei den Puppen von Formica polyctena 5 Eiweißfraktionen.

Nach Einführung von Agar-Gel als Träger der Elektrophorese wurden bis zu 10 Eiweißfraktionen oder Apoenzyme bestimmt. Velthuis et al. ${ }^{15}$ und VAN ASPERN ${ }^{16}$ stellten 7 Fraktionen Esterasen bei Musca domestica fest und MAREK ${ }^{17}$ bei den Vorpuppen von Galleria mellonella die gleiche Zahl.

Die Elektrophorese auf Stärke- und Polyacrylamidogel führte zu einer weitaus besseren Trennung der Proteine. Eine der ersten Arbeiten über Insekten finden wir bei Denucé ${ }^{18}$ und WhitTaker und WEST ${ }^{19}$, die bei verschiedenen Insektenarten bis zu 15 Eiweißbanden bestimmten. In letzter Zeit konnten LOUGHTON und WeST ${ }^{20}$, in einer Arbeit berichten, daß es ihnen gelang bei Larven, Puppen und Imagen von Malacosoma und Rotschildia bis zu 15 Banden der Hämolymphen-Proteine zu trennen. Bei Gryllus campestris $\hat{\theta}$ bestimmten MARTY und ZALTA ${ }^{21}$ gleichfalls 15 Banden und bei Weibchen 12 Banden. Bei Oncopeltus fasciatus erzielten Terando und Feir ${ }^{22}$ im besten Falle 11 Banden, von welchen 8 Banden in Richtung Kathode migrierten. MAREK ${ }^{17}$ erzielte bei den Vorpuppen von $\mathrm{Gal}$ leria mellonella bis zu 23 Proteinbanden.

${ }^{6}$ G. R. Wyatt, Ann. Rev. Entomol. 6, 75 [1961].

7 L. I. Gilbert u. H. A. Schneiderman, Amer. Zool. 1, 11 [1961].

8 J. M. Denucé, Ann. Soc. roy. Zool. Belgique 86, 151 [1955].

9 J. M. Denucé, 3. Kolloquium over Proteinen, Brügge 1955.

10 K. Aizawa, Nippon Sanshigaku Zashi. 24, 393 [1955].

11 P. S. Chen, Rev. suisse zool. 63, 216 [1956].

12 C. Wunderly u. H. Gloor, Protoplasma 42, 273 [1953].

${ }_{13}$ P. S. Chen, J. Insect Physiol. 2, 38 [1958].

14 G. H. Schmidt, J. Insect Physiol. 11, 71 [1965].

15 H. H. W. Velthuis u. K. van Aspern, Entomol. exp. appl. 6, 79 [1963].

16 K. van Aspern, J. Insect. Physiol. 8, 401 [1962].

17 M. Marek, Comparat. Biochem. Physiol., im Druck [1969].

18 J. M. DenucÉ, Z. Naturforschg. 13 b, 215 [1958].

19 J. R. Whittaker u. A. S. West, Canad. J. Zool. 40, 655 [1962].
Trotz zahlreicher, genauerer Studien über Proteine der Insektenhämolymphen stehen nur wenige Informationen über die Eigenschaften der einheitlichen Proteine zur Verfügung. Mol.-Gew.-Bestimmungen, (z. B. für das Hämoglobin von Chironomus mit 31400 ) wurden das erstemal 1934 von Svedberg und Eriksson-Quensel ${ }^{23}$ durchgeführt. Darnach folgten chronologisch Arbeiten von GLASER und STANLeY ${ }^{24}$, die bei den Hämolymphen-Larven von Bombyx mori ein Protein von verhältnismäßig hohen Mol.-Gew. 30000 (17 S) bestimmten. Auch ONCley, LeVEnBooK und Williams ${ }^{25}$ ermittelten in der Vorpuppen-Hämolymphe von Platysamia cecropia das Mol.-Gew. in 3 verschiedenen Fraktionen mit 450000 . Eine interessante Arbeit publizierte ODA ${ }^{26}$, die die Fraktionierung und physikalische Klassifikation der Proteine in der Hämolymphe von Bombyx mori behandelt. ODA ${ }^{26}$ trennte nach der Tisselius - Methode der freien Elektrophorese bei den Raupen dieser Insekten die Proteine der Hämolymphe in 3 Fraktionen, bei den Puppen in zwei Fraktionen und in der Ultrazentrifuge bestimmte er bei der 1. Fraktion das Mol.-Gew. mit $166000(2,8 \mathrm{~S})$ und bei der 2. Fraktion mit $63000(17,4 \mathrm{~S})$.

Den chromatographischen Ionenaustausch in der Zellulosesäule DEAE zur Trennung der Proteine der Hämolymphe führte erstmalig LAUFER ${ }^{27}$ durch und erhielt 3 große und 1 kleine Fraktion. Loughton ${ }^{28}$ bestimmte bei Malacosoma americana in der DEAEZellulose 5 Fraktionen und bei Rotschildia orizaba 4 Fraktionen. Das Mol.-Gew. der einzelnen Fraktionen ermittelte er auf der Ultrazentrifuge.

KRYSAN und CHADwicK ${ }^{29}$ bestimmten in der Cholinesterase des Kopfes der Musca domestica auf Sephadex G-200 ein Mol.-Gew. von 160 000, mit

20 B. G. Loughton u. A. S. West, J. Insect Physiol. 11, 919 [1965].

21 R. Marty u. J. P. Zalta, C. R. hebd. Séances Acad. Sci. Paris 263, 180 [1966].

22 S. M. L. Terando u. D. Feir, Comparat. Biochem. Physiol. 21, 31 [1967].

23 T. Svedberg u. I. Eriksson-Quensel, J. Amer. chem. Soc. 56, 1700 [1934].

24 R. W. Glaser u. W. M. Stanley, J. exp. Medicine 77, 451 [1943].

25 J. OnCley, L. Levenbook u. C. M. Williams, [1951], zitiert von K. D. Roeder, Insect Physiology, John Wiley, INC., New York 1953

26 J. ODA, Nippon Nogei-Kagaku Kaishi 30, 345 [1956].

27 H. Laufer, Ann. N. Y. Acad. Sci. 94, 825 [1961].

28 B. G. Loughton, J. Insect Physiol. 11, 1651 [1965].

29 J. L. Krysan u. L. E. Chadwick, J. Insect Physiol. 12, 781 [1966]. 
der Ultrazentrifuge erhielten sie den Wert 105000. LOUGHTON ${ }^{28}$ ermittelte in den Eiweißfraktionen der Hämolymphe von Malacosoma americana 5 Esterasefraktionen und in der von Rotschildia ori$z a b a 6$ Fraktionen. Afsharpour und O'Brien ${ }^{30}$ trennten auf der gleichen DEAE-Zellulose die Esterasen und wandten bei Periplaneta americana, Leptinotarsa decemlineata, Apis mellifica, Musca domestica, Epilachna varivestis, Oncopeltus fasciatus und Tetranychus telarius 3 Substrate zur Bestimmung von 1 bis 4 Fraktionen an, unter Berücksichtigung der Rotationsgeschwindigkeit des Homogenisators.

\section{Material und Methode}

Die Versuche wurden an Vorpuppen und Puppen der Wachsmotte Galleria mellonella (gehalten bei $30{ }^{\circ} \mathrm{C}$ in Dunkelheit und bei $60^{\circ}$ relativer Luftfeuchtigkeit) durchgeführt; als Futter diente Haydaks Kunstfutter. Die Vorpuppen und Puppen wurden in Wasser narkotisiert und ihnen dann Hämolymphe lateral mittels Einstich entnommen. Es wurde in jedes silikonisierte Reagenzglas Hämolymphe Phenylthioharnstoff als antimelanisierender Stoff beigesetzt. Die gekühlten Vorpuppen und Puppen wurden bei $4{ }^{\circ} \mathrm{C}$ durch $8-9$ Tage zwecks Bildung des Proteins bei Kälte belassen, dieses dann analysiert.

Die ligaturierten Vorpuppen wurden knapp hinter dem Kopf mit chirurgischem Zwirn unterbunden und nach 42 Stdn. wurde die Hämolymphe durch Anschneiden der Beine entnommen. Die Muster der Hämolymphe wurden bei $15000 \mathrm{U} /$ Min. zentrifugiert und das Supernatant ohne Hämocyten dan bei $-20{ }^{\circ} \mathrm{C}$ bis zum Zeitpunkt des Versuches gehalten.

Eine Säule $(1,2 \cdot 18 \mathrm{~cm})$ Sephadex G-200 (ein Geschenk des Doz. Dr. B. Gelotte, Pharmacia, Uppsala) wurde in deionisiertem Wasser $48 \mathrm{Stdn}$. gequellt und dann mit 0,03 M Phosphatpuffer bei $\mathrm{pH} 7,010 \mathrm{Stdn}$. gewaschen. Das chromatographische Röhrchen wurde mit einer $1 \mathrm{~cm}$ hohen Glaswatteschicht beschickt (statt der darin befindlichen Fritte) und über diese noch eine $1 \mathrm{~cm}$ hohe Schicht Sephadex G-25 geschichtet und erst dann gequelltes Sephadex G-200 zugesetzt. Auf die obere Schicht Sephadex wurde ein kreisrundes Papierfilter What. No. 1 vom Durchmesser $1,0 \mathrm{~cm}$ gelegt. Auf dieses Papier wurde $0,5-0,8 \mathrm{ml}$ der Hämolymphe getröpfelt. Nach dem Aufsaugen der Hämolymphe wurde auf die Oberfläche mit größter Vorsicht Phosphatpuffer zugesetzt und zwar so, daß er kontinuierlich in einer Menge, entsprechend dem Fluß der Gel-Filtration, floß. Der Zufluß bewegte sich um ungefähr $12 \mathrm{ml} / 1 \mathrm{~h}$ bei einer Fraktionsmenge von $2 \mathrm{ml} / 10$ Minuten. Die Gel-

30 F. Afsharpour u. R. D. O’Brien, J. Insect Physiol. 9, 521 [1963].

31 O. Smithies, Biochem. J. 61, 629 [1955].

32 O. Smithies, Biochem. J. 71, 585 [1959].

33 A. I. Chernoff u. N. M. Pettit, Blood 240, 750 [1964].
Filtration dauerte 5 - 6 Stunden. Es wurden innerhalb dieser Zeit $30-36$ Muster mit $60-72 \mathrm{ml}$ Eluat erzielt. Alle Lösungen wurden schon vor der Gel-Filtration mit Phenylthioharnstoff als Antimelanisations-Mittel gesättigt. Die Versuche verliefen bei Zimmertemperatur. Die einzelnen Reagenzgläser mit den filtrierten Eiweißstoffen wurden nach Beendigung der Filtration in einen Kühlraum $\left(4{ }^{\circ} \mathrm{C}\right)$ gebracht. Die Muster der einzelnen Reagenzgläser wurden bei $280 \mathrm{~m} \mu$ des Beckmanschen Spektrophotometers gemessen. Bei einigen Mustern wurde auch ihr Absorptionsspektrum im automatischen Spektrophotometer OPTICA, Milano, bestimmt.

Die Aktivität der Esterase wurde nach der Methode von Afsharpour et al. ${ }^{30}$ ermittelt.

Der Rest des Eluates wurde im Vakuum in $35 \mathrm{Stdn}$. konzentriert und zwar mittels Luftpumpe über Cellophanpapier. $70 \mu \mathrm{l}$ des verdickten Musters wurden auf eine vertikale Stärkegelelektrophorese nach Smithies ${ }^{31,32}$ übertragen. Die Puffer für das Gel und die Wannen enthielten Borsäure, TRIS, Chelaton 2 und Chelaton 3, wie sie erstmals von CHERnoff und PetTit ${ }^{33}$ verwendet wurden. Die Stärkegelelektrophorese wurde bei $4{ }^{\circ} \mathrm{C}, 600 \mathrm{~V}$ und einem Potentialgefälle von $15 \mathrm{~V} / \mathrm{cm}$ durchgeführt. Nach 4 bis $5 \mathrm{Stdn}$. Elektrophorese wurde das Stärkegel der Länge nach in $3(2 \mathrm{~mm}$ dicke) Schichten zerschnitten und eine von ihnen zur Identifizierung der Eiweißstoffe mit Amidoschwarz $10 \mathrm{~B}$ gefärbt. Die andere Schicht wurde zur Bestimmung der Esterasen 1 Stde. in 1-proz. Acetonlösung von 1-Naphthylbutyrat in Phosphorpuffer $0,2 \mathrm{M}, \mathrm{pH}$ 6,7 belassen. Die Diazotierungsreaktion des 1-Naphthylbutyrates als Substrat der Esterasen verlief bei Zimmertemperatur und bei Kopulation mit Fast Blue BB-Salz. Die 3. Schicht des Stärkegels wurde 30 Min. in eine Lösung Phosphatpuffer von $\mathrm{pH} 6,7$ und $1 \cdot 10^{-4} \mathrm{M}$ Eserin eingelegt, die die Entstehung aller Banden von Cholinesterase verhindert. Die nach der Inhibition verbliebenen Banden reihen wir in die Arylesterasen ein. Die ausgewerteten Stärkeschichten wurden dann zwecks Konservierung in Gelatine (Gelatina animalis), Glycerin und Wasser nach DANGERFIELD und FAULKNER ${ }^{34}$ plastifiziert.

Das Mol.-Gew. der Eiweißstoffe in der Hämolymphe der Vorpuppen und Puppen von Galleria mellonella wurde mittels Gel-Filtration auf dünner Schicht Sephadex G-200, Superfine ( vis Marek ${ }^{35}$ ) bestimmt. Die Proben, in welchen das Mol.-Gew. der Eiweißstoffe ermittelt wurde, entnahm man den konzentrierten Eluaten der Säulen der Gel-Filtration der Peake A, B (vis Abbn. 1-5) .

Die Bestimmung des isoelektrischen Punktes der fraktionierten Proteine geschah mittels Papierelektrophorese. Die Pufferlösungen von verschiedenem $\mathrm{pH}$ wurden nach der Methode von Miller und Golder ${ }^{36}$ hergestellt. Die Papierelektrophorese verlief $4 \mathrm{Stdn}$. bei

34 N. G. Dangerfield u. G. Faulkner, Nature [London] 200. 388 [1963].

35 M. Marek, Experientia [Basel] 25, 136 [1969].

36 L. G. Miller u. H. R. Golder. Arch. Biochem. 29, 420 [1950]. 
$4{ }^{\circ} \mathrm{C}$, das Potentialgefälle war $10 \mathrm{~V} / \mathrm{cm}$. Das Protein, das nach $4 \mathrm{Stdn}$. Elektrophorese am Start blieb, hat einen isoelektrischen Punkt, der dem Wert jenes $\mathrm{pH}$ Puffers entspricht, der in der Wanne der Elektrophorese angewandt wurde.

Zwecks Elimination eventuell störend wirkender elektroendosmotischer Einflüsse wurde am Start als Indikator Blue-Dextran 2000 verwendet.

\section{Ergebnisse}

Der erste Versuch der Gel-Filtration auf Sephadex G-200 wurde mit 0,8 ml Hämolymphe der Vorpuppe Galleria mellonella durchgeführt. Das Ergebnis dieser Filtration waren 2 Peaks nach Messung aller Reagenzgläser mit Eluaten bei $280 \mathrm{~m} \mu$. Von
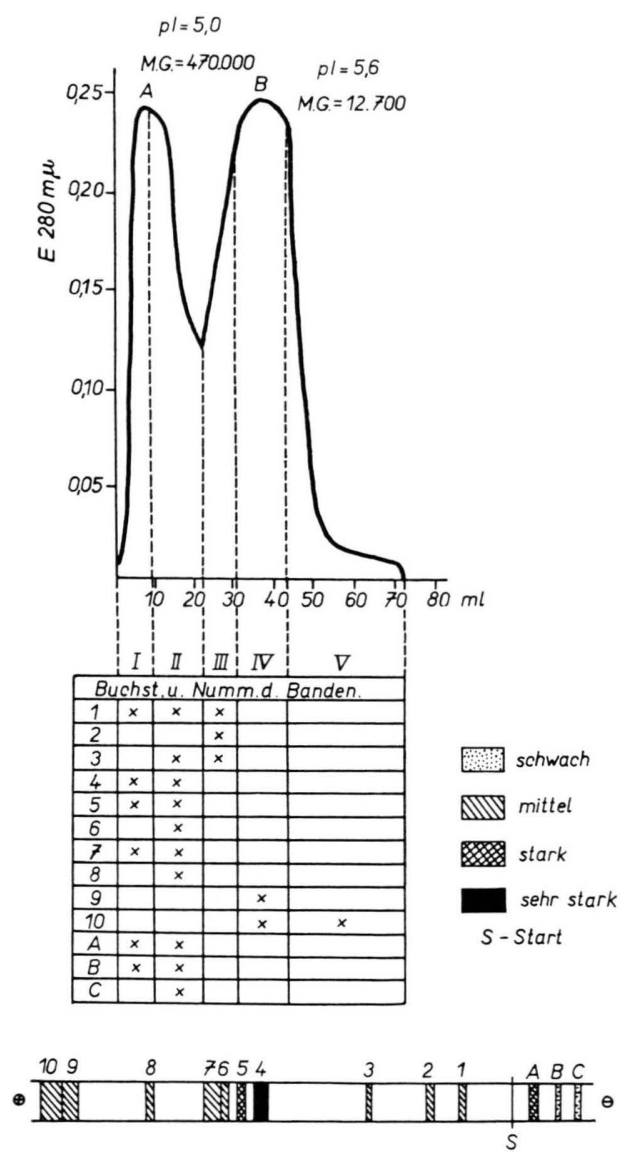

Abb. 1 a. Die Gel-Filtration der Hämolympheproteine der Vorpuppen von Galleria mellonella. Die Proteinaktivität bestimmt bei $280 \mathrm{~m} \mu$. Die konzentrierten Eluate der Zonen I-V wurden auf Stärkegel mittels Elektrophorese getrennt. Die Proteinbanden wurden mit einem Kreuz bezeichnet und entsprechend den elektrischen Ladungen unter Nummer des Elektropherogramms der nichtfiltrierten Hämolymphe eingereiht.

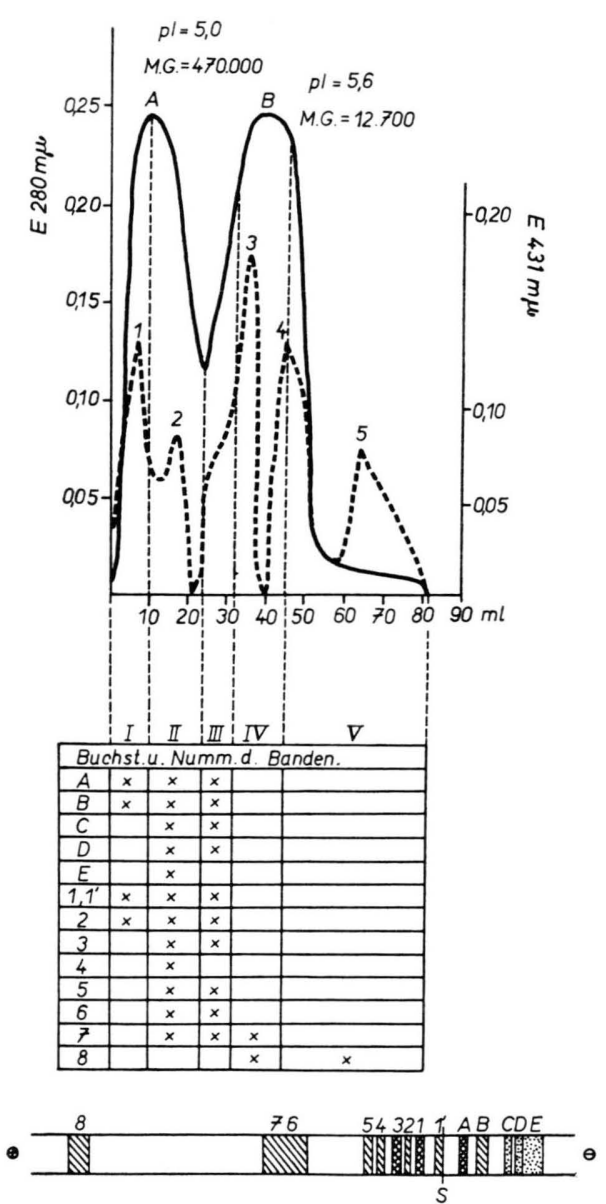

Abb. l b. Die Gel-Filtration der Hämolympheproteine der Vorpuppen von Galleria mellonella. Die Aktivität der Esterase ( --() , bestimmt bei $431 \mathrm{~m} \mu$ und die Proteinaktivität bei $280 \mathrm{~m} \mu$ (-). Die konzentrierten Eluate der Zonen I-V getrennt mittels Stärkegelelektrophorese. Die aufscheinenden Esterasebanden wurden mit Kreuz bezeichnet und entsprechend der elektrischen Ladung nach Nummern und Buchstaben in das Elektropherogramm der nichtfiltrierten Hämolymphe eingereiht.

den verdickten Eluaten wurden Proben erzielt, die auf Stärkegelelektrophorese der einzelnen Zonen I - V aufgetragen wurden. So wurde eine Übersicht erzielt, wie die einzelnen Eiweißbanden, erhalten mittels Elektrophorese auf Stärkegel, filtriert durch das Molekularsieb Sephadex, in Abhängigkeit ihres Mol.-Gew., verlaufen. In Peak A $(p I=5,0)$, Abb. 1 a, erschienen meist Eiweißstoffe von höherem Mol.Gew., als in Peak B von $p \mathrm{I}=5,6$. Die meisten durch Stärkegelelektrophorese getrennten Eiweißstoffe zeigten sich regelmäßig in Peak A der Zone II.

Durch Gel-Filtration wurde das Apoenzym der Esterase, bestimmt mittels $\beta$-Naphthylacetat in 5 
Gipfel getrennt (vis Abb. 1 b), von denen das konzentrierte Eluat II, der Gipfel 2, aufgetragen auf Stärkegelelektrophorese durch Elektrophorese in 14 Esterasebanden getrennt wurde. Das Eserin inhibierte die Entstehung der Banden 7 und 8, die wir in die Cholinesterasen einreihten. Die übrigen Banden gehörten den Arylesterasen und Aliesterasen an.

Der Versuch einer Gelfiltration der Vorpuppen (mittels Ligatur erdrosselt), wurde ähnlich durchgeführt, wie der vorstehend ausgeführte Versuch. Die Zahl der in Stärkegelelektrophorese getrennten Eiweißstoffe erhöhte sich vor allem im Peak C

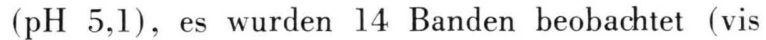
Abb. 2 a). Aus der Abb. 2 b ist zu ersehen, daß in der Zone I im Gipfel 2 ein „Ligaturprotein“ (X) bestimmt wurde, das in die Arylesterasen oder Aliesterasen einzureihen ist. Bei der Elektrophorese wanderte es zur Kathode und wurde mit dem Buch-
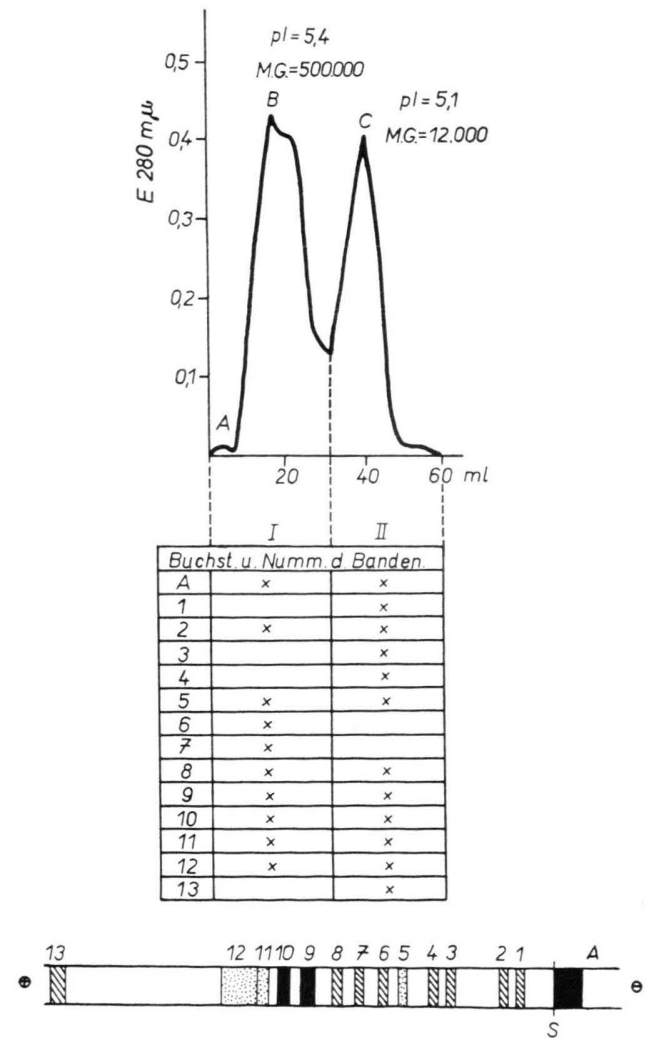

Abb. 2 a. Die Gel-Filtration der Hämolymphe der ligaturierten Vorpuppen von Galleria mellonella. Die Proteinaktivität bestimmt bei $280 \mathrm{~m} \mu$. Die konzentrierten Eluate der Zonen I-II wurden auf Stärkegel mittels Elektrophorese getrennt. Die Proteinbanden wurden mit einem Kreuz bezeichnet und entsprechend den elektrischen Ladungen unter Nummer des Elektropherogramms der nichtfiltrierten Hämolymphe eingereiht.

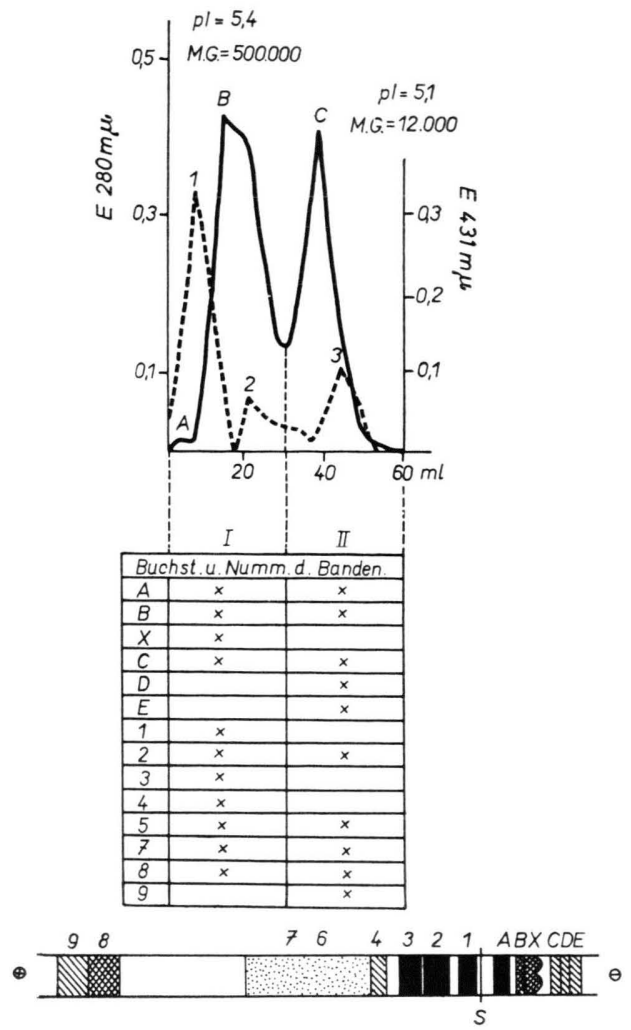

Abb. 2 b. Die Gel-Filtration der Hämolymphe der ligaturierten Vorpuppen von Galleria mellonella. Die Aktivität der Esterase $(---)$, bestimmt bei $431 \mathrm{~m} \mu$ und die Proteinaktivität bei $280 \mathrm{~m} \mu$ (-). Die konzentrierten Eluate der Zonen I-II getrennt mittels Stärkegelelektrophorese. Die aufscheinenden Esterasebanden wurden mit Kreuz bezeichnet und entsprechend der elektrischen Ladung nach Nummern und Buchstaben in das Elektropherogramm der nichtfiltrierten Hämolymphe eingereiht. „X“ $=$ ligaturiertes Protein.

staben „X“ bezeichnet. Bei Vorpuppen ohne Anwendung von Ligatur zeigte sich kein "Ligaturprotein“ und auch nicht bei der weiteren Entwicklung der Puppen und des Imagos.

Schließlich wurden Versuche einer Gelfiltration bei der Hämolymphe männlicher Puppen durchgeführt, desgleichen solche mit unterkühlten Puppen bei $4{ }^{\circ} \mathrm{C}$. Man erhielt 2 Peaks (vis Abbn. 3 a, 3 b und Abbn. 4 a, 4 b). In der Hämolymphe der Weibchenpuppen wurden 3 Hauptpeaks erhalten, von welchen Peak B am reichhaltigsten an Eiweißbanden auf dem Stärkegel war und ebenso reichhaltig an Esterasen (vis Abbn. 5 a, 5 b) .

Der isoelektrische Punkt der Eiweißstoffe, bestimmt bei Vorpuppen und Puppen, zeichnete sich dadurch aus, daß die Fraktion A fast bei allen Versuchen einen um einige Zehntel höheren $p I$ aufwies 

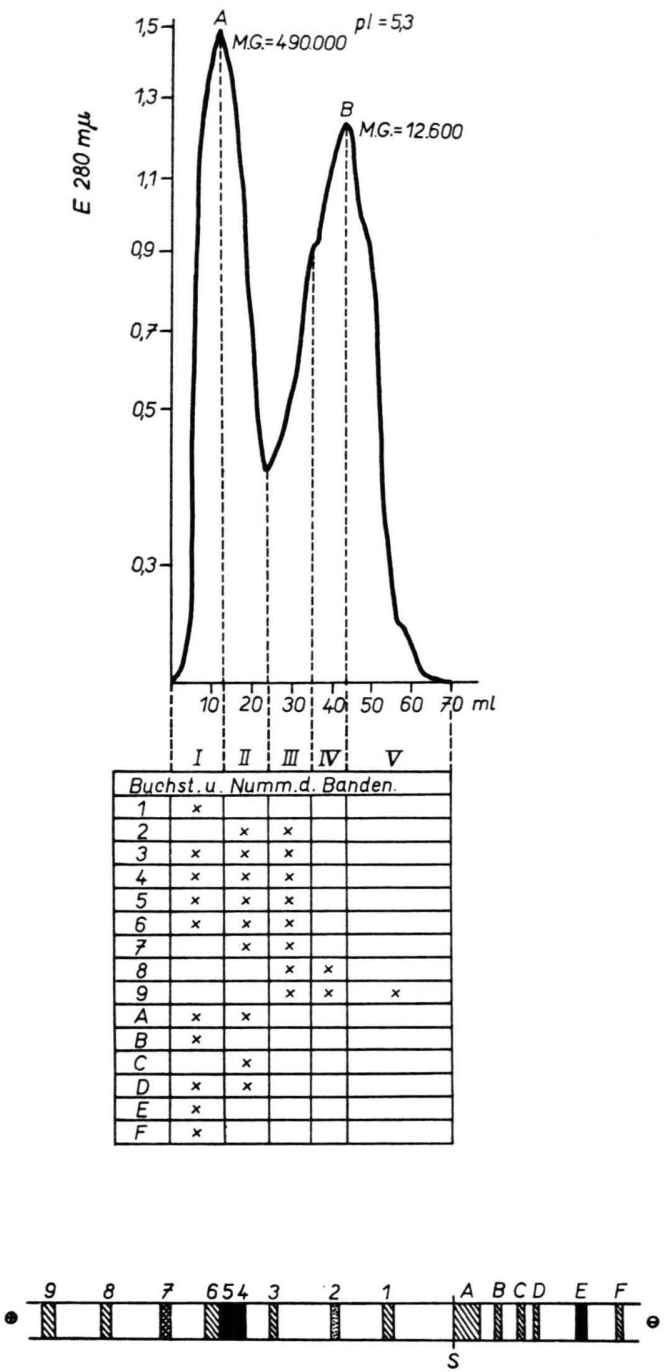

Abb. 3 a. Die Gel-Filtration der Hämolympheproteine der männlichen Puppen von Galleria mellonella. Proteinaktivität bestimmt bei $280 \mathrm{~m} \mu$. Die konzentrierten Eluate der Zonen I-V wurden auf Stärkegel mittels Elektrophorese getrennt. Die Proteinbanden wurden mit einem Kreuz bezeichnet und entsprechend den elektrischen Ladungen unter Nummer des Elektropherogramms der nichtfiltrierten Hämolymphe eingereiht.

als die Fraktion B. Der isoelektrische Punkt aller Eiweißfraktionen bewegte sich von $p I=5,0$ bis $p \mathrm{I}=5,6$ (vis Abbn. $1 \mathrm{a}, 1 \mathrm{~b}$ bis $5 \mathrm{a}, 5 \mathrm{~b}$ ) .

\section{Diskussion}

Obgleich zahlreiche Arbeiten über die Trennung der Proteine der Insektenhämolymphe durch Elektrophorese und Immunodiffusion auf Agar publiziert
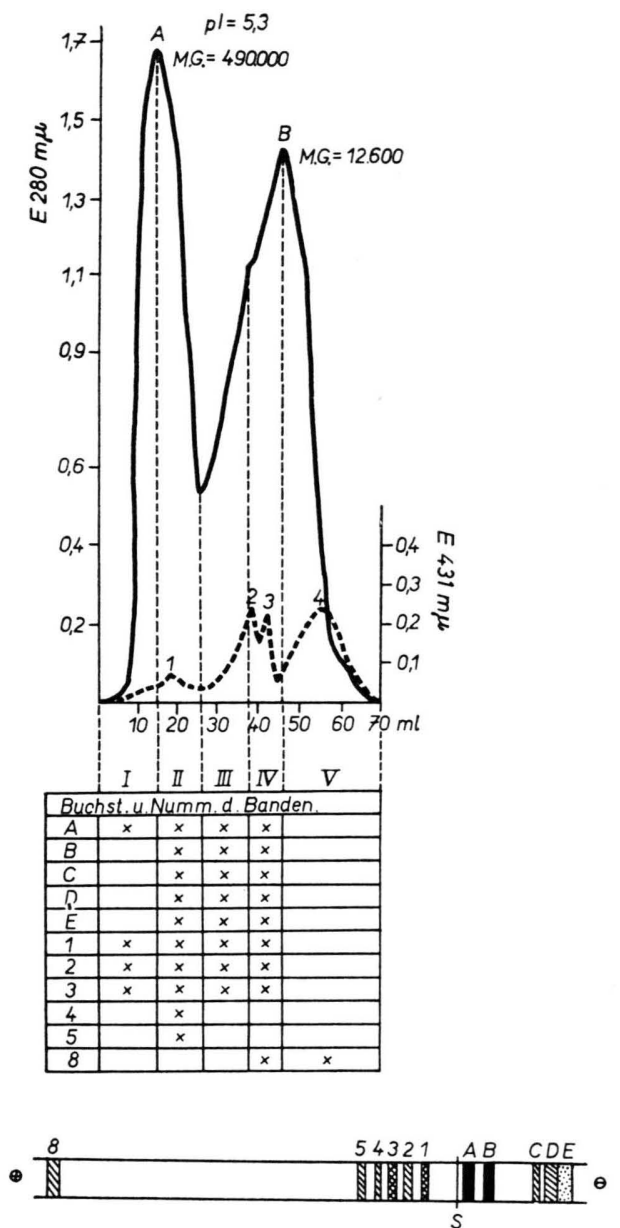

Abb. 3 b. Die Gel-Filtration der Hämolympheproteine der männlichen Puppen von Galleria mellonella. Die Aktivität der Esterase (- - ), bestimmt bei $431 \mathrm{~m} \mu$, und die Proteinaktivität bei $280 \mathrm{~m} \mu$ (-). Die konzentrierten Eluate der Zonen I-V getrennt mittels Stärkegelelektrophorese. Die aufscheinenden Esterasebanden wurden mit Kreuz bezeichnet und entsprechend der elektrischen Ladung nach Nummern und Buchstaben in das Elektropherogramm der nichtfiltrierten Hämolymphe eingereiht.

wurden, existiert noch kein allgemeines System einer Nomenklatur zu ihrer Beschreibung. Das liegt an der ungenügenden Information über ihre Funktion und Charakteristik. Die vorliegende Arbeit will sich wenigstens teilweise bemühen, den Versuch zu unternehmen, die Proteine von Vorpuppen und Puppen der Galleria mellonella auf Grund der Gelfiltration und mit Hilfe der Stärkegelelektrophorese zu trennen; überdies auch einige Hauptcharakteristika der fraktionierten Eiweißstoffe (den isoelektrischen Punkt und das Mol.-Gew.) zu bestimmen. 

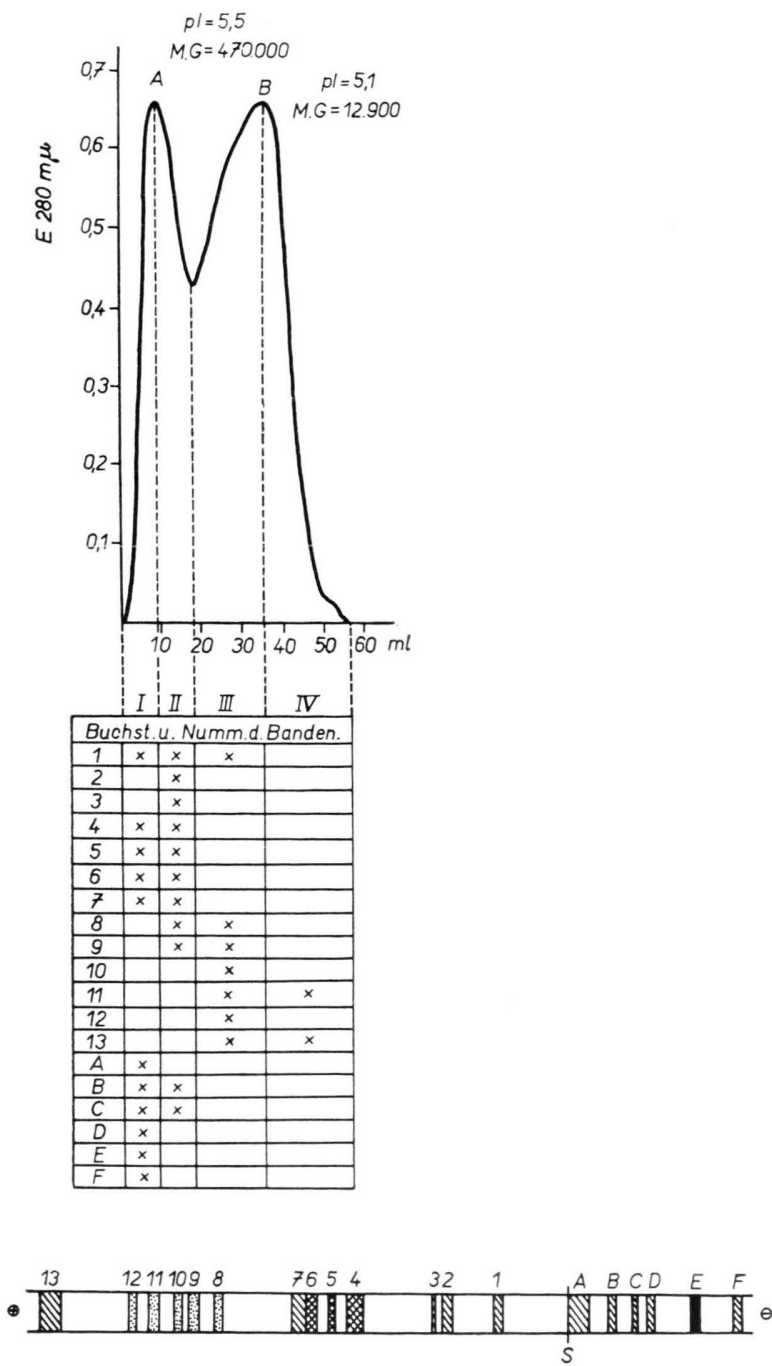

Abb. 4 a. Die Gel-Filtration der Hämolympheproteine unterkühlter Puppen von Galleria mellonella. Die Proteinaktivität bestimmt bei $280 \mathrm{~m} \mu$. Die konzentrierten Eluate der Zonen I-IV wurden auf Stärkegel mittels Elektrophorese getrennt. Die Proteinbanden wurden mit einem Kreuz bezeichnet und entsprechend den elektrischen Ladungen unter Nummer des Elektropherogramms der nichtfiltrierten Hämolymphe eingereiht.

Mit einer ähnlichen Thematik befaßte sich bereits LOUGHTON ${ }^{28}$, der für die Eiweißtrennung der Hämolymphe, DEAE-Zellulose verwendete, die, wie sich zeigte, sich günstiger für die Trennung in mehrere Fraktionen erwies. Sephadex G-200 erreicht zwar die Vorzüge von DEAE-Zellulose nicht, trotzdem gestatten konzentrierte Proben, entnommen einigen Zonen des gleichen Peaks der Gelfiltration, zwecks elektrophoretischer Trennung, qualitative und quantitative Unterschiede in den Proben festzustel-

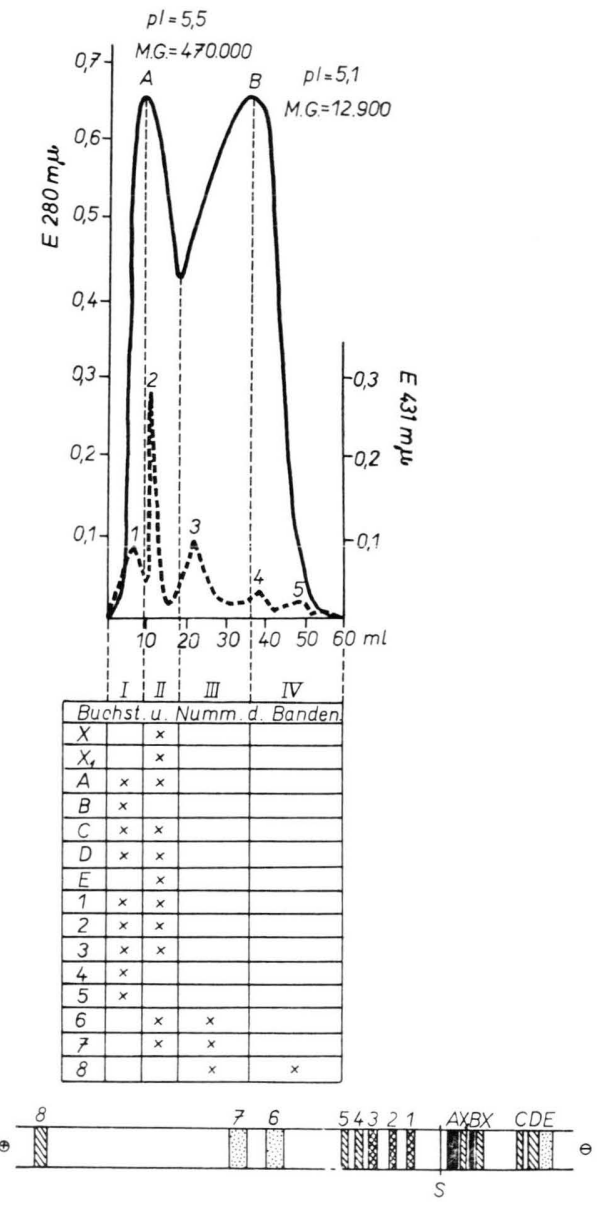

Abb. 4 b. Die Gel-Filtration der Hämolympheproteine unterkühlter Puppen von Galleria mellonella. Die Aktivität der Esterase (- - ), bestimmt bei $431 \mathrm{~m} \mu$, und die Proteinaktivität bei $280 \mathrm{~m} \mu(-)$. Die konzentrierten Eluate der Zonen I-IV getrennt mittels Stärkegelelektrophorese. Die aufscheinenden Esterasebanden wurden mit Kreuz bezeichnet und entsprechend der elektrischen Ladung nach Nummern und Buchstaben in das Elektropherogramm der nichtfiltrierten Hämolymphe eingereiht. „, $\mathrm{X}^{“}=$ Unterkühlungs-Protein.

len. Das Interesse für Insektenesterasen steigerte sich sowie sich das Interesse für den Metabolismus der Insekticiden erhöhte. Menzel, Craig und Hoskins ${ }^{37}$ vergleichen die Esterasen resistenter und wenig resistenter Stämme von Musca domenstica und Afsharpour und $\mathrm{O}^{\prime}$ Brien $^{30}$ wandten die Chromatographie des Ionenaustausches zur Fraktionierung der Insektenesterasen bei 7 Insektenarten an. Obwohl diese Autoren Homogenate des Körpergewebes verwendeten und LougHTON ${ }^{28}$ bei Malacosoma americana die Hämolymphe, sind ihre Ergebnisse, erzielt nach der chromatographischen Methode des Ionenaustausches, sehr ähnlich. 

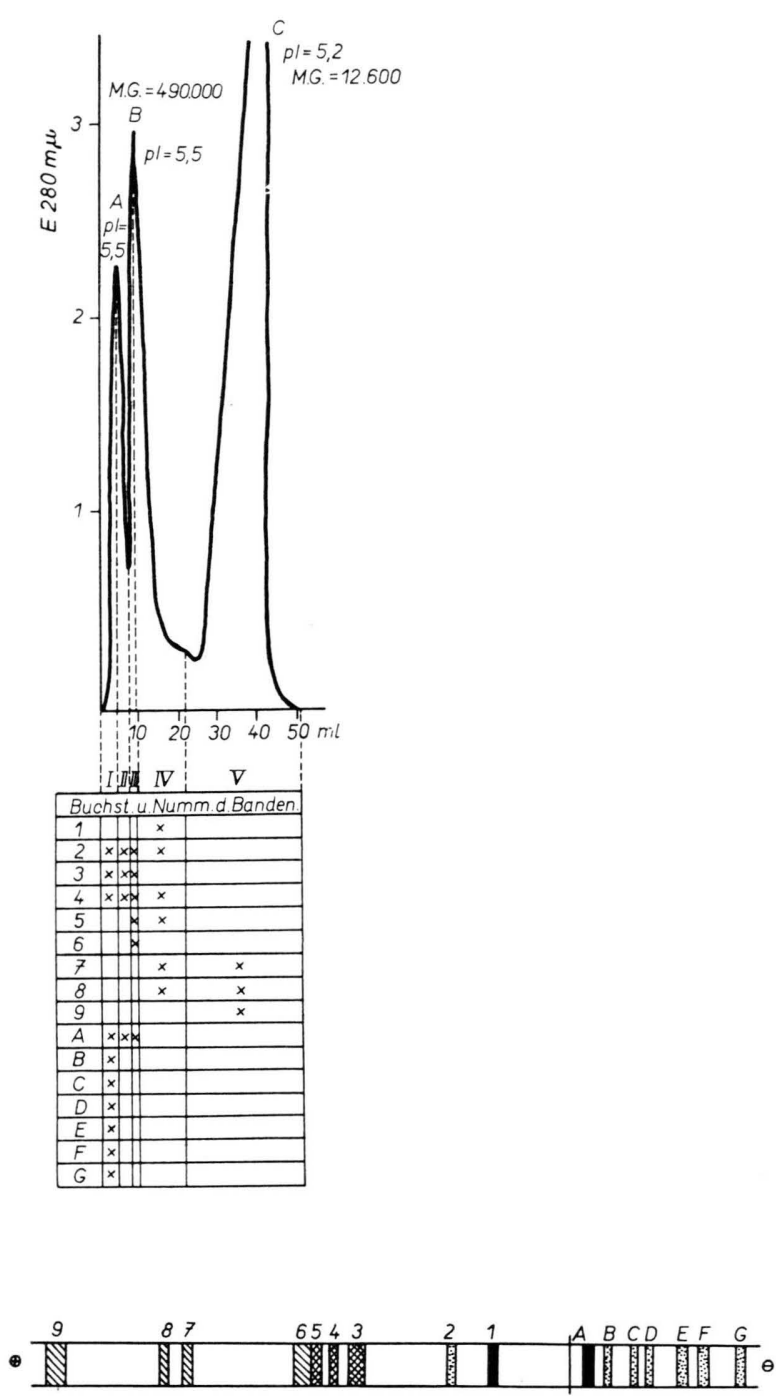

Abb. 5 a. Die Gel-Filtration der Hämolympheproteine der Puppen vom Weibchen Galleria mellonella. Die Proteinaktivität bestimmt bei $280 \mathrm{~m} \mu$. Die konzentrierten Eluate der Zonen $\mathrm{I}-\mathrm{V}$ wurden auf Stärkegel mittels Elektrophorese getrennt. Die Proteinbanden wurden mit einem Kreuz bezeichnet und entsprechend den elektrischen Ladungen nach Nummer und Buchstaben in das Elektropherogramm der nichtfiltrierten Hämolymphe eingereiht.

Die Trennung der Esterasen mittels Gelfiltration war günstiger als die der DEAE-Zellulose-Trennung, was ad 1. die 5 aus der Gelfiltration auf Sephadex G-200 erzielten Gipfel und ad 2. der Nachweis der Existenz von „Protein gekühlter" aus unterkühlten Puppen (vis Abb. 4 b, "X“) und ad 3. des "Ligaturproteins" (bei, durch Ligatur gedrosselter Vorpuppen bestimmt), mittels Elektrophorese in kon-
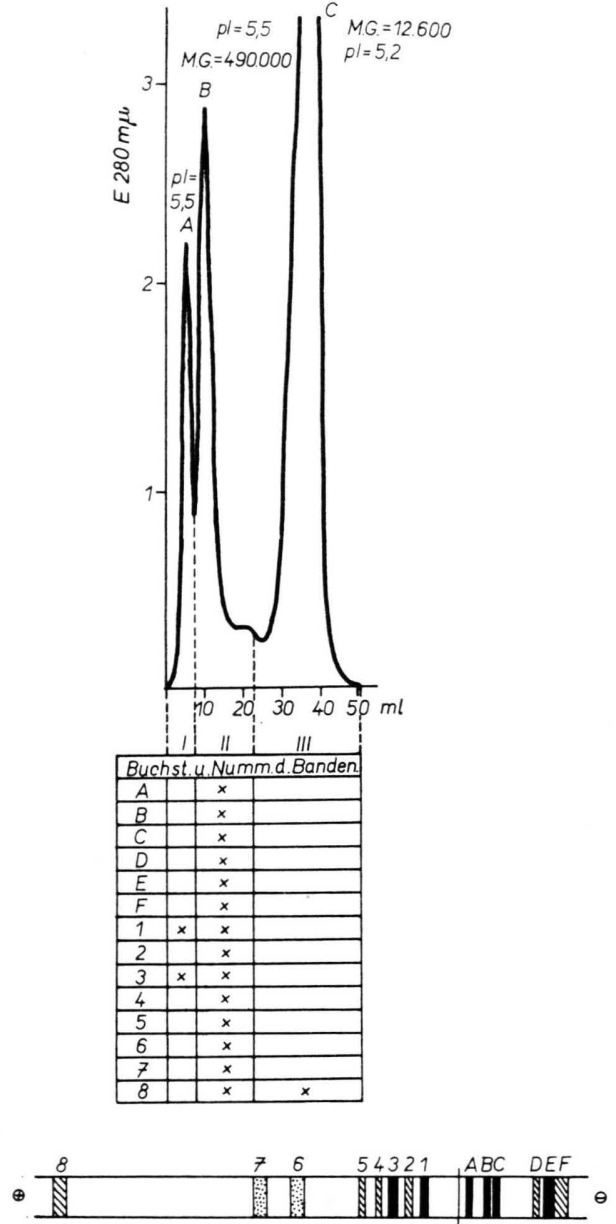

Abb. 5 b. Die Gel-Filtration der Hämolympheproteine der Puppen vom Weibchen Galleria mellonella. Die Proteinaktivität bestimmt bei $280 \mathrm{~m} \mu$. Die konzentrierten Eluate der Zonen I-III wurden auf Stärkegel mittels Elektrophorese getrennt. Die aufscheinenden Esterasebanden wurden mit Kreuz bezeichnet und entsprechend der elektrischen Ladung nach Nummern und Buchstaben in das Elektropherogramm der nichtfiltrierten Hämolymphe eingereiht.

zentrierten Eluaten, bewiesen (vis Abb. 2 b, „X“).

Die Werte für die isoelektrischen Punkte, bestimmt für die einzelnen Peaks, der mittels Gelfiltration getrennter Proteine bei Galleria mellonella, stimmen fast vollkommen mit jenen von LOUGHTON 28 angeführten Werten überein. Interessant ist die Tatsache, daß Peak A bis auf eine Ausnahme bei weiblichen Puppen, einen um einige Zehntel höheren $p I$ Wert besitzt als Peak B. Diese Ergebnisse lassen sich mit den Arbeiten anderer Autoren schwer ver-

37 D. Menzel, R. Craig u. W. M. Hoskins, J. Insect. Physiol. 9, 479 [1963]. 
gleichen, da an diesem Problem bei Insekten wenig gearbeitet wurde. Bemerkenswert ist die Arbeit von Bladergroen $^{38}$ über den isoelektrischen Punkt, bestimmt bei Plasmaeiweißstoffen des Menschen, ermittelt nach der Papierelektrophorese: Albumin $-p I=4,64 ; \quad \alpha$-Globulin $\quad-p I=4,8 ; \quad \beta$-Globulin

38 W. Bladergroen, Physikalische Chemie in Medizin und Biologie, S. 354, Basel 1949. $-p I=5,2 ; \quad$ Fibrinogen $\quad-p \mathrm{I}=5,4 ; \quad \gamma$-Globulin $-p I=6,4$. Aus den angeführten Werten ist ersichtlich, daß nur das $\beta$-Globulin einen ähnlichen Wert $p I$ hat, wie dieser für die Proteine der Hämolymphe bei Vorpuppen und Puppen von Galleria mellonella bestimmt wurde und wie ihn LOUGHTON ${ }^{28}$ bei $M a$ lacosoma americana und Rotschildia orizaba ermittelte.

\title{
Über Zusammenhänge zwischen chemischer Struktur und herbizider Wirkung bei Chinazolin-Derivaten und verwandten heterocyclischen Systemen
}

\author{
Adolf Zeidler, Adolf Fischer und Günter Scheuerer \\ Hauptlaboratorium und Landwirtschaftliche Abteilung der Badischen Anilin- und Soda-Fabrik, \\ Ludwigshafen/Rhein \\ (Z. Naturforschg. 24 b, 740-744 [1969]; eingegangen am 3. Februar 1969)
}

Herrn Professor Dr. B. Tiмm zum 60. Geburtstag gewidmet

\begin{abstract}
In den 3-Alkyl-2.4-dioxo-tetrahydrochinazolinen wurde eine neue Klasse herbizider Wirkstoffe gefunden. Die herbizide Wirkung der Chinazolindione ist an das Vorhandensein der PyrimidinStruktur des Heterocyclus gebunden und hängt ferner von den Substituenten an den beiden Pyrimidin-Stickstoffatomen $\mathrm{ab}$ - sie wird gesteigert, wenn der ankondensierte Benzolring zum Cyclohexanring hydriert ist. Durch Veränderungen am Heterocyclus durch Einführung anderer Heteroatome resultierten wirkungslose Verbindungen oder aber im Falle der Benzo-2.1.3-thiadiazinon-4.2.2-dioxide eine neue herbizide Wirkstoffklasse, deren Wirkungsmechanismus jedoch völlig verschieden von dem der Chinazolindione ist.
\end{abstract}

Vor etwa 20 Jahren wurde der $N$-p-Chlorphenyl$N^{\prime}, N^{\prime}$-dimethylharnstoff ${ }^{1}$ als Herbizid entwickelt. In der Folgezeit wurde der Einfluß der verschiedenen Substituenten am Harnstoffmolekül auf die herbizide Wirksamkeit untersucht, und auch Verbindungen geprüft, welche die Harnstoffgruppierung als Teil eines heterocyclischen Ringsystems enthielten.

So wurden in der Klasse der Uracile stark wirksame Herbizide aufgefunden, von denen die Totalmittel ${ }^{\circledR}$ Hyvar $(\mathbf{1} \mathbf{a})$ und ${ }^{\circledR}$ Bromacil $(\mathbf{1} \mathbf{b})$ der Firma Du Pont genannt seien ${ }^{2}$.<smiles>[R]n1c(=O)[nH]c(C)c(Br)c1=O</smiles><smiles>CC(=[18O])C(C)C</smiles>

Eigene Arbeiten über Harnstoff-Derivate der Anthranilsäure, die keine besondere herbizide Wirk-

1 US-Pat. 2665445 (1952), Du Pont de Nemours (CH. W. TODD).

2 Deutsche Auslegeschrift 1240698 (1960) DuPont de Nemours (L. M. Ellis, H. M. Loux, R. W. Luckenbaugh u. E. J. SовоcZenski). samkeit zeigten, führten zur Körperklasse der 2.4-Dioxo-tetrahydro-chinazoline ${ }^{3}(2)$, die chemisch den Uracilen verwandt sind. Im Gegensatz zu den herbiciden Harnstoffen hängt die Wirksamkeit der Uracile 1 von dem Vorhandensein kleiner aliphatischer Reste am Pyrimidin-Stickstoff in 3-Stellung ab. Es sollte deshalb geklärt werden, ob sich diese Beobachtung auch auf die 2.4-Dioxo-tetrahydro-chinazoline (2) übertragen läßt.

Für die Synthese von Chinazolindionen finden sich in der Literatur eine Reihe von Angaben. So reagiert Isatosäureanhydrid mit Alkylisocyanaten in siedendem Dimethylformamid unter Bildung von 3-Alkyl-2.4-dioxo-tetrahydrochinazolinen ${ }^{4}$. Beim Zusammenschmelzen von Anthranilsäure mit Phenylharnstoff wird 3-Phenyl-2.4-dioxo-tetrahydrochinazolin erhalten ${ }^{5}$.

\footnotetext{
${ }^{3}$ Deutsches Bundes-Patent 1210242 (1964) BASF (G. Scheuerer, A. Zeidler u. A. Fischer).

4 R. P. Staiger, C. L. Mayer u. G. R. Picher, J. Chem. Eng. Data 8, 454 [1963].

5 F. S. Spring u. J. C. Woods, J. chem. Soc. [London] $1945,625$.
} 